\title{
Norois
}

Environnement, aménagement, société

$205 \mid 2007 / 4$

Vivre les espaces périurbains

\section{La mobilité du quotidien dans les espaces périurbains, une grande diversité de modèles de déplacements}

L'exemple de la métropole nantaise

Daily mobility in peri-urban spaces, a great diversity of movements patterns. An example: the Nantes - Saint-Nazaire metropolitan area

\section{Vincent Hervouet}

\section{(2) OpenEdition}

1 Journals

\section{Édition électronique}

URL : https://journals.openedition.org/norois/2073

DOI : 10.4000/norois. 2073

ISBN : 978-2-7535-1553-6

ISSN : $1760-8546$

\section{Éditeur}

Presses universitaires de Rennes

Édition imprimée

Date de publication : 1 décembre 2007

Pagination : $37-52$

ISBN : 978-2-7535-0616-9

ISSN : 0029-182X

\section{Référence électronique}

Vincent Hervouet, "La mobilité du quotidien dans les espaces périurbains, une grande diversité de modèles de déplacements », Norois [En ligne], 205 | 2007/4, mis en ligne le 01 décembre 2009, consulté le 13 janvier 2022. URL : http://journals.openedition.org/norois/2073 ; DOI : https://doi.org/ 10.4000/norois. 2073 


\title{
LA MOBILITÉ DU QUOTIDIEN DANS LES ESPACES PÉRIURBAINS, UNE GRANDE DIVERSITÉ DE MODÈLES DE DÉPLACEMENTS L'EXEMPLE DE LA MÉTROPOLE NANTAISE
}

\author{
Vincent HeRVOUET \\ CESTAM - UMR ESO 6590 CNRS \\ (Université de Nantes), \\ Chemin de la Censive-du-Tertre, BP 81227 - 44312 NANTES cedex 03 \\ vincent.hervouet@wanadoo.fr
}

\section{RÉSUMÉ}

Depuis les années soixante, la périurbanisation a progressivement remis en cause le modèle européen de la ville dense. En effet, l'étalement urbain se fait, non plus en contiguïté avec les espaces bâtis existants, mais par dissémination de zones résidentielles pavillonnaires dans les espaces périphériques. Cet éloignement géographique est possible grâce à l'usage massif et quotidien de l'automobile. L'étude des déplacements dans la métropole Nantes - Saint-Nazaire, à travers la mobilité des ménages de douze communes périurbaines, révèle qu'il existe une grande diversité de modèles de déplacements. S'organisant selon des rapports très variés à la ville (permanent, grandissant, pragmatique, relayé, contraint, naissant) ils présentent chacun des caractéristiques particulières qui montrent toute la diversité des rapports à l'espace dans les espaces périurbains

Mots CLÉ : Espaces périurbains - Étalement urbain - Ménages - Métropole NantesSaint-Nazaire - Mobilité du quotidien - Modèles de déplacements.

\section{ABSTRACT}

Daily mobility in peri-urban spaces, a great diversity of movements patterns. An example: the Nantes - Saint-Nazaire metropolitan area

Since the 1960s, periurbanization has progressively called into question the European model of a compact city. Indeed, urban sprawl no longer progresses in contiguity with existing developments, but by the dissemination of private housing zones in peripheral spaces. This geographic distancing is possible thanks to the massive and daily use of the automobile. Research on this movements in the metropolitan area of Nantes - Saint-Nazaire, through the mobility of households from twelve periurban cities, reveals that there are, indeed, an important number of mobility patterns. Depending on the case, these mobility patterns can take place in a permanent, increasing, pragmatic, relayed, rejected, or emerging relationship and that's why they have each ones their own particularities revealing all the diversity of spatial dynamics in the peri-urban spaces.

KEY WORDS : Daily Mobility - Households - Mobility Pattern - Nantes - Peri-urban spaces - Nantes-Saint-Nazaire Metropolitan Areas - Urban Sprawl. 
Depuis les années d'après-guerre, la forme urbaine s'est profondément métamorphosée : après une période de forte croissance due à l'exode rural, le flux démographique s'est inversé avec l'apparition de la périurbanisation autour des villes européennes. Consacrant l’installation de populations urbaines dans les campagnes, ce processus présente la particularité de se réaliser en discontinuité spatiale avec la ville (Bauer, Roux, 1976) : la continuité du bâti ne constitue plus un critère suffisant pour s'assurer d'être toujours dans la ville; des portions s'en détachent géographiquement mais peuvent conserver des relations avec elle sans que cela s'inscrive dans le paysage. L'urbain devient ainsi beaucoup plus difficilement saisissable. Les interrogations se multiplient quant à la nature de son évolution. Que devient-il en périphérie? Se transforme-t-il en un autre objet, ni tout à fait urbain ni tout à fait rural ? Une urbanité périphérique, coupée de ses racines citadines, est-elle possible?

L'approche de la périurbanisation par le biais des acteurs individuels que sont les ménages périurbains s'impose tant l'individu apparaît central dans le processus, qu'il prenne réellement des décisions selon son libre-arbitre ou qu’il subisse des mécanismes politiques, économiques, sociaux, géographiques le dépassant. Les phénomènes de mobilité qui sont associés à l'individu ou à la cellule familiale matérialisent bien sûr son inscription territoriale - ce qui constitue déjà un premier intérêt - mais surtout constituent, à l'échelle de l'histoire urbaine, le facteur de nouveauté qui doit être saisi pour mieux connaître les rouages de la ville étalée. Ils apparaissent donc comme une clef de lecture pertinente des espaces périurbains.

Nantes, l'une des dix plus grandes villes de France, constitue un terrain d'étude répondant à tous les critères qu'exige une telle problématique : son poids démographique lui permet de disposer d'une banlieue agglomérée importante et d'espaces périurbains s'étendant sur plusieurs dizaines de kilomètres; par ailleurs, la métropole qu'elle forme avec Saint-Nazaire s'insère quasi intégralement dans un département - la Loire-Atlantique - où coexistent espaces urbains, périurbains et ruraux aux évolutions contrastées. Grâce à la réalisation de 400 questionnaires complétés par 30 entretiens auprès de particuliers de douze communes périurbaines nantaises et nazairiennes ${ }^{1}$, la mobilité du quotidien de ces populations périurbaines a pu être saisie dans son intégralité. Cet article se propose ainsi d'exposer trois conclusions fortes de ces recherches de terrain. Tout d'abord, l'analyse de la relation entre l'usager et son automobile montre l'importance de ce moyen de locomotion dans les espaces périurbains. Ensuite, les parcours de vie (passé et présent) des habitants périurbains ont une grande incidence sur leur façon de se déplacer. Enfin, des modèles de déplacement sont identifiables quant à l'organisation générale des déplacements des populations périurbaines. Si ces modèles ne permettent pas à eux seuls une vision complète des déplacements dans les espaces périurbains, l'étude de la mobilité résidentielle devant être réintroduite dans une analyse plus globale, ils constituent une approche importante des phénomènes de mobilité en marge des villes.

\section{L'habitant périurbain et son automobile : entre intimité, liberté et contrainte}

Comprendre les mécanismes de la mobilité du quotidien dans les espaces périurbains, c'est d'abord cerner le rapport complexe qui unit l'habitant périurbain à un outil indispensable à ses déplacements, sa voiture. Cette nécessité d’appréhender la mobilité autrement que sous le seul

1. Un échantillon de douze communes périurbaines (au sens INSEE du terme) tenant compte de la diversité démographique et du positionnement par rapport à la ville voisine a été choisi. 400 questionnaires déposés en boîte à lettres et récupérés par voie postale, en porte-à-porte ou en mairie y ont été réalisés auprès de particuliers propriétaires vivant dans les lotissements construits depuis une quarantaine d'années dans ces communes. 30 entretiens (auprès de personnes déjà enquêtées par questionnaires) respectant la diversité de l'échantillon de base ont permis un approfondissement des thématiques explorées (situation, mobilité résidentielle, mobilité du quotidien). Les analyses qui nourrissent cet article sont ainsi issues du recoupement de ces deux sources, la première étant d'ordre plus quantitatif et la seconde plus qualitative (bien que cette distinction soit à nuancer). 
angle de la description des possibilités de déplacements ou des migrations effectives est défendue, notamment, par V. Kaufmann et B. Montulet (2004). Dans la même perspective, J.-P. Orfeuil (1994) et G. Dupuy (1999) mettent en avant les liens d'attachement, d'appropriation voire d'identification entre l'usager et sa voiture. Plus qu'un simple objet utilitaire et de consommation, la voiture constitue pour ses passagers un outil de liberté, d'action individuelle ou familiale - notamment au vu de la déficience des transports collectifs en milieu périurbain - mais aussi une part de la sphère privée au même titre que le logement. La relation véhicule/utilisateur(s) se révèle riche de sens.

\section{LA NATURE DE LA RELATION VOITURE/USAGER}

L'analyse des discours des ménages ${ }^{2}$ sur l'usage de l'automobile permet de mieux comprendre ce qui relie le conducteur et ses passagers au véhicule automobile utilisé.

Chaque automobiliste prend différemment possession de l'espace intérieur de son véhicule. Le niveau de personnalisation de l'habitacle est ainsi un indicateur du degré d'appropriation au même titre que l'aménagement du domicile, lieu d'intimité familiale par excellence. Le refus de marquer cet espace par la présence d’objets personnels est quasi général. Mais les ménages interrogés prennent possession des lieux par l'ambiance qu'ils y (re)créent : écouter l'autoradio ou de la musique, converser avec les autres passagers ou au contraire, "éviter le dialogue ", " apprécier le silence », « rêvasser », ou encore « organiser son emploi du temps ».

Soumis à des déplacements quotidiens de plus en plus longs et fréquents (Bonnet, Desjeux, 2000), le conducteur et ses éventuels passagers pourraient exprimer la montée d'un mécontentement général sur le vécu des «systèmes individuels de mobilité » (Ascher, 1995, p. 202). Or, malgré le contexte de mise à distance résidentielle, les temps de trajet sont intégrés comme une nécessité ce qui les rend ipso facto supportables. L'automobile peut même constituer un lieu de refuge à l'écart des trépidations de la vie familiale. Ainsi l'aspect fatigant et pénible de ces " pérégrinations ${ }^{3}$ » est assez peu souligné par les personnes interrogées.

Si l'achat raisonné de l'objet utilitaire (fiabilité, sécurité, consommation, volume intérieur) prévaut globalement sur le « coup de cœur », le confort intérieur du véhicule est l'aspect qui divise le plus les usagers, entre ceux qui sont soucieux de leur bien-être personnel (et attirés par le moyen ou haut de gamme) et ceux qui affirment haut et fort le contraire (en se tournant vers le bas de gamme). L'association « petite voiture » (pour trajets courts)/ grosse voiture » (pour trajets familiaux plus longs) est souvent adoptée comme une forme d'arbitrage.

Sur le partage du véhicule avec des tierces personnes, rares sont les personnes qui manifestent un attachement à leur voiture au point de ne pas vouloir le partager avec leur conjoint. Après celuici, les autres membres proches de la famille sont les principaux emprunteurs potentiels. Le cercle des amis est plus sujet à controverse (selon le capital confiance accordé) pour le prêt du véhicule. Le covoiturage récolte l'adhésion de peu de conducteurs, sauf en cas d'extrême nécessité.

L'attachement de l'automobiliste à son véhicule relève donc avant tout du pragmatisme. C'est d'abord un objet utilitaire nécessaire aux populations périurbaines, qu’il s'agit d'acquérir en fonction de ses capacités financières, puis de conserver dans un bon état et aussi longtemps que possible. La voiture n’apparaît pas comme une pièce supplémentaire du domicile familial qu’il faudrait aménager. Par contre, c'est clairement un morceau de propriété privée où l'intimité est préservée et où un tiers non familier n'est accepté que ponctuellement.

\footnotetext{
2. Discours saisis en entretiens avec les particuliers.

3. «Le pérégrination est, pour l'habitant, un mode de gestion de la multiplicité des déplacements, lié à l'éclatement du territoire et du mode de vie, fondé sur la combinaison optimale des parcours et des arrêts entre des destinations dont l'un des termes est inévitablement la maison » (Pinson, Thomann, p. 23).
} 


\section{L'ÉMANCIPATION PAR L’AUTOMOBILE : LE SENTIMENT DU CONDUCTEUR}

Liberté, indépendance, mobilité, souplesse, flexibilité, précision, autonomie, commodité, absence de contraintes sont des notions que les automobilistes mettent en avant pour décrire les qualités qu'ils reconnaissent à leurs pratiques individuelles de mobilité. L'analyse de discours révèle que c'est avant tout la capacité de s'accommoder au mieux des impératifs de la vie quotidienne qui guide l'ensemble de ces jugements. Ainsi, l'émancipation de la contrainte horaire, le sentiment d'adopter un mode de locomotion adapté aux besoins de la famille, l'affranchissement vis-à-vis des conditions météorologiques ou de la présence d'autrui sont des arguments souvent développés. Plus classiquement, l'efficacité et la qualité de la desserte sont mis en avant alors que les transports en commun sont peu prisés en raison du délai d'attente à la gare, du rejet de la promiscuité, du manque d'indépendance, de la lenteur du car ou du risque de grève, ou tout simplement en raison de l'absence de desserte.

La propension est très nette à déplorer les désagréments individuels subis en voiture et, au contraire, à se montrer plus évasif et moins convaincu quant aux nuisances collectives occasionnées et aux mesures pour y remédier. Ralentissements, encombrements, embouteillages et difficultés de stationnement arrivent au premier rang des désagréments dont le trafic automobile est dit porteur, que ce soit de façon très ponctuelle ou quotidienne. Par contre, la prise de conscience d'enjeux plus globaux pour la société est beaucoup plus faible de la part des automobilistes; l'insouciance ou l'égoïsme prévalent même parfois. Ainsi, face aux menaces croissantes de pollution qui pèsent sur l'environnement des espaces urbains et périurbains, au mieux se dégage un sentiment général d'impuissance et de fatalité, au pire est exprimé un désintérêt total. Quant au partage des responsabilités, certains désignent d'autres acteurs plus à même, selon eux, d'agir positivement en la matière (hommes politiques, État, constructeurs automobiles, etc.). En définitive, beaucoup de ménages reconnaissent implicitement vouloir conserver leur véhicule en attendant que la société propose d'autres alternatives financièrement acceptables.

Un relatif consensus autour du «tout automobile » se dégage. Dans cet exercice d'équilibriste qui consiste à ménager l'intérêt individuel tout en respectant le bien collectif, les automobilistes semblent surtout donner priorité à la vie familiale et à tout ce qui peut faciliter son organisation. À leur décharge, l'usage de la voiture requiert un investissement financier qui légitime sans doute le droit à la mobilité selon leurs propres règles.

\section{L'USAGE DE L'AUTOMOBILE : UN INVESTISSEMENT FINANCIER À ASSUMER}

En France, la voiture mobilise environ $15 \%$ du budget des ménages (Ascher, 1995). Dans les espaces périurbains, cette charge s'ajoute à celle, déjà lourde, de l'accession à la propriété et de l'entretien de la résidence principale. Interrogés sur leur budget, les ménages des communeséchantillon ont accepté de détailler les différentes facettes de ces dépenses : leur description, leur gestion, leur évolution et leurs conséquences.

Si quelques familles assurent connaître les frais occasionnés par la possession d'une automobile - carburant, assurance, réparations, stationnement, prix d'acquisition, etc. -, nombreuses sont celles qui, soit par crainte du résultat du calcul, soit par résignation devant des dépenses inévitables, soit par l'assurance d'un niveau de revenus suffisant, n’en ont pas une idée précise.

La hausse du prix du carburant est considérée comme un fait dont il faut s'accommoder. Si beaucoup de conducteurs s'accordent sur la possibilité de réduire les frais de garagiste, en effectuant eux-mêmes les réparations de base, la réduction des déplacements comme source d'économie est rarement envisagée du fait des obligations familiales et professionnelles. C'est souvent même une augmentation de la mobilité du quotidien que les ménages doivent gérer après installation dans les espaces périurbains.

Contrairement à une hypothèse initiale relevant du registre de la contrainte, l'importance des trajets - en distance et en temps - ne semble pas aussi mal vécue par les ménages que cela. 
L'analyse de l'opinion des ménages sur leurs déplacements quotidiens (consignée dans les questionnaires et détaillée en entretiens) aboutit à un tableau nuancé : dans notre panel de ménages, les représentations positives et négatives apparaissent dans des proportions comparables. Si la qualité des infrastructures routières empruntées influe beaucoup sur ce jugement, le vécu des pérégrinations - entre l'affirmation franche d'un bien-être, la reconnaissance d'un comportement raisonné ou la dénonciation d'une mobilité lourde à assumer - semble aussi tributaire de la capacité du conducteur à prendre du recul et à s'accommoder ou non de sa situation.

Au final, l'automobilité qui marque fortement les espaces périurbains est une donnée qui s’impose aux ménages. L'éloignement géographique, la faiblesse et le manque de performance des transports en commun, le développement des infrastructures routières contribuent à attribuer à la voiture un rôle central et essentiel dans la mobilité périurbaine. Intégrant ce facteur, les automobilistes tentent de s'accommoder au mieux de cette contrainte en lui trouvant des vertus de liberté (« je circule quand et où je veux »), d'intimité ( flexibilité («je suis réactif par rapport à tout changement dans mon emploi du temps»). Dans ce rapport ambivalent de séduction/rejet pour ce moyen de locomotion, les ménages cherchent donc généralement à optimiser l'efficacité de leurs déplacements.

\section{Les principaux facteurs déterminant l'organisation des déplacements dans les espaces périurbains}

L'optimisation des déplacements dans les espaces périurbains, au-delà des arbitrages et contraintes qui agissent sur les populations, doit aussi tenir compte de trois effets de structure importants.

Tout d'abord, la nature des migrations « domicile-travail » constitue une colonne vertébrale autour de laquelle se greffe une grande partie des pérégrinations des actifs. Ensuite, l'intensité de la polarisation exercée par le pôle urbain voisin ${ }^{4}$ impulse un fort mouvement centripète aux déplacements des populations périurbaines. Enfin, les attaches conservées avec le passé résidentiel constituent des points d'ancrage que les individus continuent à fréquenter.

\section{L'IMPACT STRUCTURANT DES MIGRATIONS « DOMICILE-TRAVAIL »}

Contrairement aux déplacements très ponctuels liés à la satisfaction d'un besoin rare, les migrations contraintes liées au travail constituent un axe structurant incontournable autour duquel l'ensemble des déplacements de la personne, et par ricochets de son ménage, doit s'organiser. Sur ces déplacements, se greffent de plus en plus d'autres étapes de pérégrinations ou de déplacements en boucle (Chalas, 2004), qui peuvent modifier les schémas classiques de fréquentation.

Ainsi, bien que la toute puissance de l'automobile dans les espaces périurbains, la diversité du profil des communes-échantillon et des ménages interrogés soient, a priori, des obstacles à la quête d'une cohérence dans les pratiques individuelles ou familiales de mobilité, le lieu de travail apparaît très structurant. Quatre aires de fréquentation aux caractéristiques bien distinctes et correspondant à autant de types de communes de travail - la commune de résidence, une commune alentour distante de vingt kilomètres au plus, une commune de la banlieue agglomérée, la ville-centre - peuvent, de ce point de vue, être individualisées.

Ainsi, travailler dans sa propre commune de résidence (celle-ci est alors la plupart du temps un pôle d'emploi de taille relativement importante) implique souvent de fréquenter de préférence les équipements locaux. Seuls les déplacements dans des grandes surfaces commerciales pour des besoins ponctuels s'inscrivent dans la zone d'attraction du pôle urbain voisin. 
De même, travailler dans une commune proche du lieu de résidence tend à diriger plus facilement les déplacements vers une petite unité urbaine périphérique voisine (souvent chef-lieu de canton) que vers le grand pôle urbain voisin.

À l'opposé, les lieux préférentiels de fréquentation ${ }^{5}$ d'un adulte travaillant dans la banlieue du pôle urbain se situent nettement dans cette même banlieue, à travers l'usage des commerces de proximité, de la grande surface à usage hebdomadaire ou ponctuel et des galeries marchandes que l'on peut y trouver.

Enfin, travailler dans la ville-centre implique souvent qu'en dehors de la scolarité des enfants, qui se déroule classiquement dans la commune de résidence pour l'école primaire, les autres formes récurrentes de déplacements se font à destination du pôle urbain et, plus précisément, de la ville-centre.

La diversité des quatre types de migrations «domicile-travail » est révélatrice de l'hétérogénéité des espaces périurbains mais aussi de l'impact de la localisation des bassins d'emploi sur les dynamiques périurbaines. Sur ce point, deux systèmes de fonctionnement se concurrencent et se complètent : les pôles périurbains d'emplois parviennent à fixer sur place une partie des populations résidantes et tentent de résister à une deuxième logique à savoir la forte concentration métropolitaine des activités dans la ville-centre et dans les zones d'activités industrielles et commerciales de l'agglomération.

Ainsi, les ménages sont tiraillés entre des logiques résidentielles d'enracinement local, auxquelles ils aspirent d'ailleurs souvent, et des évolutions économiques qui attisent et augmentent sans cesse les flux centripètes de travailleurs vers le pôle urbain voisin.

\section{LA FORCE DE LA POLARISATION URBAINE}

Dépositaire d'une offre rare que leur importance démographique permet de concentrer, les grands pôles urbains drainent une clientèle dans un rayon de plusieurs dizaines de kilomètres alentours. Ce type d'offre répond ainsi aux besoins non satisfaits dans les espaces périurbains. C'est principalement le niveau supérieur de l'offre médicale ou commerciale qui se concentre ainsi dans les agglomérations urbaines. Dans cette répartition territoriale, l'ensemble Nantes - Saint-Nazaire fait donc valoir son rôle de métropole régionale en offrant l'accès à des services et des commerces rares aux populations des deux aires urbaines.

En effet, grâce à leur réseau de cliniques et d’hôpitaux complété par une myriade de médecins spécialistes disséminés dans les deux pôles urbains, les agglomérations nantaise et nazairienne proposent une offre médicale sans équivalence dans un rayon de cent kilomètres.

De même, les établissements scolaires de l'enseignement supérieur et du second degré du secondaire (lycées, BTS, IUP, IUT, grandes écoles, universités...) participent à l'attractivité de la métropole nantaise; seuls les collèges et les écoles élémentaires relèvent encore (jusqu’à la fin de la carte scolaire?) de l'échelon communal ou multicommunal.

Concurrençant ou relayant les moyennes et grandes surfaces présentes dans les petits pôles urbains périphériques (Jousseaume, 1998), les « grandes surfaces » alimentaires ou spécialisées de l'agglomération nantaise offrent une gamme plus étendue de produits, notamment sur les produits de consommation moins courante. Répondant donc à des besoins ponctuels ou spécifiques (aquariophilie, ameublement, jardinage, etc.), l'achat de ces produits occasionne globalement des déplacements peu nombreux - mensuels, pluriannuels voire parfois seulement annuels, mais toujours très polarisés.

La fréquentation des commerces et services du centre de Nantes est du domaine, par contre, de l'agrément ou s'émancipe des contingences du quotidien. En effet, bien que souvent lié à un besoin précis - un objet décoratif rare, un accessoire précis pour l'équipement de la maison, une

5. Définis par les réponses les plus liées à une caractéristique particulière de la personne - en l'occurrence le lieu de travail - à partir de quelques indices statistiques de base (écart à l'indépendance, khi² et pourcentage de l'écart maximum). 
tenue de mariage, etc. -, la fréquentation du centre constitue souvent une occasion de promenade, de flânerie dans les rues du centre-ville. Les ménages mettent ainsi en évidence l'aspect ponctuel et récréatif de cette «sortie en ville».

La richesse de l'offre culturelle (cinémas, théâtres, concerts, etc.) est également l'une des raisons qui attirent dans le centre de Nantes les populations périurbaines. L'aspect non contraint de ces déplacements donne une signification sociale forte à ce type de fréquentation : venir dans un centre-ville nantais peu accessible à l'automobile relève d'un attachement au cadre citadin et d'un plaisir à le fréquenter. Malgré la résidence périurbaine, le lien avec la « ville compacte » peut demeurer solide.

\section{LE RÔLE IMPORTANT DU PASSÉ RÉSIDENTIEL DES MÉNAGES}

Le parcours résidentiel laisse également des «traces » sur la façon actuelle de se déplacer. Des corrélations peuvent être faites entre la commune de résidence quittée et la nature des déplacements des ménages après leur installation dans les espaces périurbains. Ces remarques prennent un relief particulier quand le parcours résidentiel a fait étape dans la ville-centre et dans sa banlieue agglomérée avant une migration en périphérie. Contrairement aux déplacements contraints déjà étudiés - pour des besoins alimentaires, médicaux ou scolaires - les destinations « choisies » semblent tenir compte de cette antériorité.

Ainsi, l'observation des déplacements des ménages pour rendre visite à leur famille met en évidence le rôle de la localisation de la commune de résidence précédente. L’hypothèse de travail selon laquelle l'installation dans les espaces périurbains peut constituer une importante «mise à distance » d'un milieu de vie familier et assez largement investi durant les années précédant le déménagement, est à nuancer : comme la parentèle y demeure toujours, elle constitue un point d'ancrage important et récurrent pour le ménage périurbain.

À l'instar des visites à la famille, les déplacements pour voir des amis apparaissent fortement influencés par le lieu de résidence ayant précédé celui du logement actuel : lorsqu'il s'agit d'effectuer des visites amicales, les ménages ont une plus grande propension à fréquenter le type de communes où ils ont demeuré lors de l'étape résidentielle précédente. Les liens noués quelques années précédemment demeurent souvent, même après l'éloignement géographique que représente l'installation dans les espaces périurbains, en particulier pour les anciens citadins nantais ou nazairiens.

Quant aux loisirs des adultes périurbains, le comparatif entre la commune de résidence précédente et les lieux de fréquentation aboutit à la même conclusion que pour les deux premiers types de déplacements cités : le lieu de résidence quitté reste proportionnellement plus fréquenté pour les loisirs par les ménages qui y demeuraient auparavant que par l'ensemble des familles du panel. Les habitudes prises en matière de loisirs durant l'étape résidentielle précédente semblent donc perdurer partiellement malgré le changement de commune de résidence.

Au final, les ménages périurbains restent très influencés dans leurs déplacements par le travail, la proximité de la ville et leur passé résidentiel : les choix qu'ils estiment faire pour leurs déplacements en sont tributaires.

\section{Les différents modèles de déplacements du quotidien des populations périurbaines}

Malgré la complexité des déplacements observés dans les espaces périurbains, il est possible de mettre en évidence plusieurs modèles de déplacements du quotidien. Au-delà des variations interindividuelles, interfamiliales et intercommunales, six modèles se distinguent. Idéaux-types synthétisant une multitude de situations particulières intermédiaires, ces modèles permettent de sérier les différentes logiques qui sont à l'œuvre chez les populations périurbaines, selon la nature 
du rapport qu'elles entretiennent avec la ville voisine. Pour illustrer chacun de ces modèles, un exemple significatif de déplacements d'un ménage interrogé par nos soins a été retenu et présenté, carte à l'appui. Ces déplacements ont été décrits par les intéressés lorsque nous avons interrogé successivement et thématiquement ces derniers sur leurs trajets professionnels ou récréatifs, leurs visites aux amis ou à la famille, etc. Les cartes, dans un souci de clarté, n’intègrent pas les combinaisons de déplacements et donc la notion déjà expliquée de pérégrination.

\section{PREMIER MODÈLE : UN RAPPORT PERMANENT À LA VILLE}

Dans ce modèle, nombreuses sont les raisons de déplacements au quotidien vers la ville, au-delà du lieu de travail. Aux attaches familiales et amicales passées, se greffent assez classiquement les courses alimentaires en grande surface, la fréquentation des galeries marchandes et des médecins spécialistes; par contre, de façon plus originale, l'offre en commerces et services relativement étoffée de la commune de résidence n'empêche pas une part importante des ménages de se déplacer dans le pôle urbain voisin pour profiter des commerces de proximité, d'un médecin généraliste, d'une agence bancaire, d'un garage automobile ou d'un salon de coiffure. Les activités de loisirs des chefs de famille, des conjoints et des enfants, n'échappent pas à cette attirance permanente pour le pôle urbain nantais ou nazairien. Cela ne signifie pas pour autant que la commune de résidence ne soit pas fréquentée lors des multiples activités de la vie quotidienne. Dans le cas où celle-ci est chef-lieu de canton, elle a tout de même quelques atouts à faire valoir même si l'agglomération nantaise - voire parfois une unité urbaine voisine - réduit son influence. Le rapport permanent à la ville chez ces habitants se perçoit aussi par le fait qu'ils se disent soit « urbains », soit « périurbains », soit « semi urbains ».

Les déplacements d'un ménage vivant à Aigrefeuille-sur-Maine (fig. 1) soulignent qu'en dehors de la commune de résidence, la quasi-totalité des pérégrinations se dirigent vers le pôle urbain voisin. Seuls les parcours pour rendre visite à la famille ou aux amis paraissent échapper partiellement à cette logique radiale.

\section{DEUXIÈME MODÈLE : UN RAPPORT GRANDISSANT À LA VILLE}

Ce modèle se retrouve souvent chez des populations périurbaines vivant dans des communes longtemps moins soumises à l'influence urbaine ${ }^{6}$ que dans le premier modèle, bien qu'à distance comparable de la ville centre. Les déplacements actuels au quotidien de ces ménages sont de plus en plus dirigés vers le pôle urbain voisin.

Certes, la commune de résidence contente la majorité des besoins en commerces et services de proximité. Néanmoins, une grande part des ménages effectue également des pérégrinations dans la ville-centre ou dans sa banlieue pour ces mêmes fins. Les courses alimentaires, la fréquentation des galeries marchandes, la consultation de médecins spécialistes se réalisent de manière quasi exclusive dans le pôle urbain. Mais l'importance des relations amicales et familiales qui y ont cours est révélatrice des liens étroits qui unissent ce type de ménages à l'agglomération voisine. Seule l'auto-définition identitaire de ces populations laisse entrevoir que la diffusion de l'urbanité en ces lieux n'a pas encore atteint le stade décrit pour le modèle précédent : ici les habitants revendiquent plus le qualificatif de « semi-rural » que celui de « semi-urbain »; l'affirmation du caractère « urbain » ou « périurbain » recueille, par ailleurs, encore assez peu de suffrages. Le changement de contexte local est encore assez récent dans les mentalités et la population « de souche », encore nombreuse, continue à jouer un rôle de garant des valeurs locales traditionnelles empreintes de ruralité, ce qui freine la diffusion d'un fort rapport à la ville.

6. Souvent en raison d'un gel de l'urbanisation décidé par des édiles municipaux sous la pression des propriétaires terriens locaux soucieux de conserver leur patrimoine foncier. 


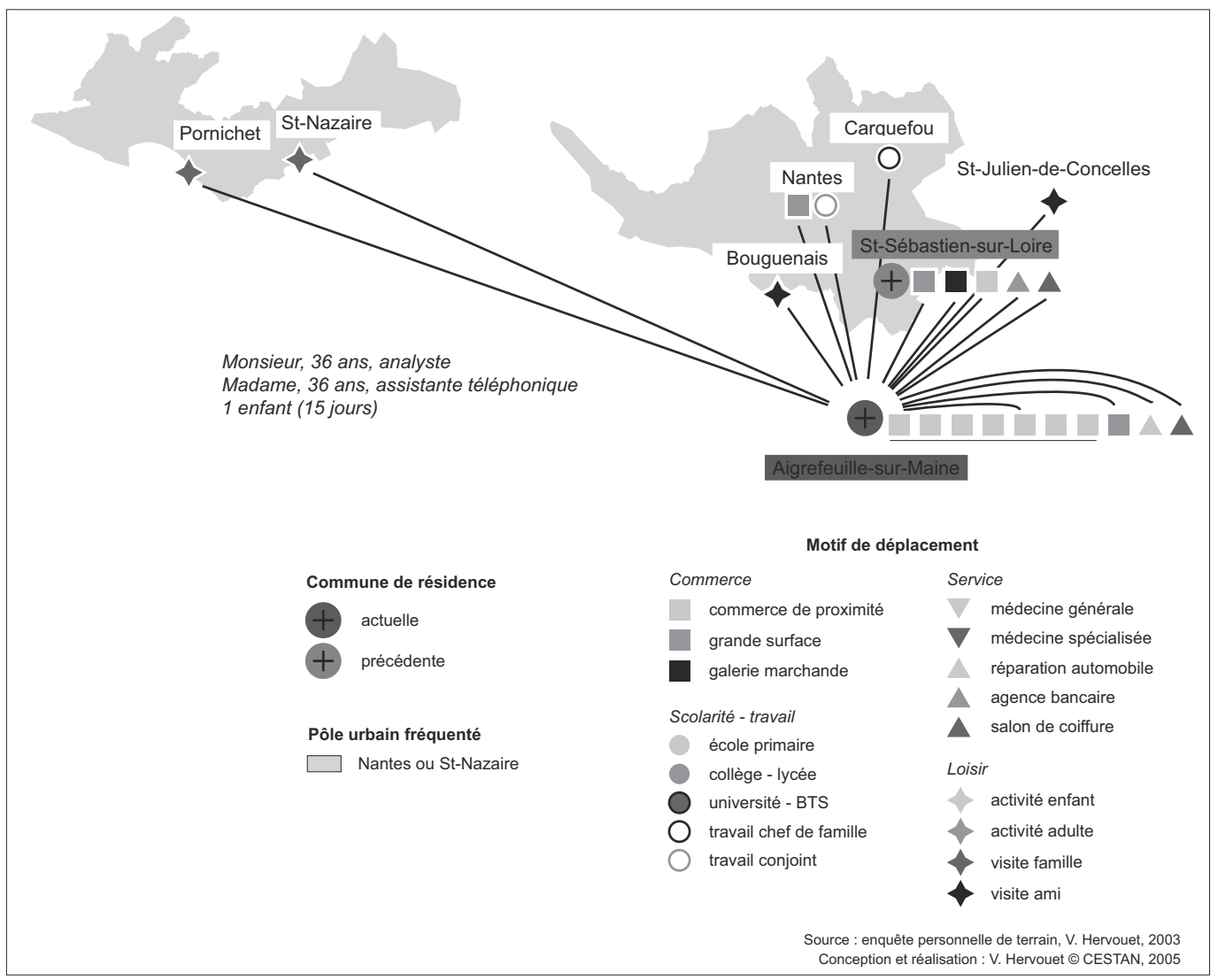

Figure 1 : Exemple de déplacements d'un ménage : le modèle du rapport permanent à la ville An example of household mobility : the "permanent relationship with town" pattern

Ceci est illustré par l'orientation urbaine des déplacements d'un ménage de cinq personnes à Mauves-sur-Loire (fig. 2). Hormis les besoins basiques de cette famille (commerces et services de proximité, écoles et activités de loisirs pour les enfants), les autres déplacements s'effectuent vers et dans le pôle urbain voisin : les deux conjoints y travaillent, y font leurs courses, déposent leur enfant au collège, y ont leur banque et y pratiquent leurs activités; le week-end est aussi le moment d'y retourner pour fréquenter famille et amis.

\section{TROISIÈME MODÈLE : UN RAPPORT PRAGMATIQUE ET DISTANCIÉ À LA VILLE}

Souvent aussi plus éloignées du pôle urbain, les actifs entrant dans ce modèle sont moins nombreux à travailler dans l'agglomération nantaise. Soucieux de vivre dans un cadre de vie à caractère champêtre, ces ménages n'ont recours qu'avec parcimonie à la fréquentation de la ville voisine et préfèrent contenter leurs besoins en services et commerces de proximité dans la commune de résidence même. Étant donné l'éloignement géographique de la ville-centre, des unités urbaines périphériques voisines, grâce à leur offre commerciale, médicale et économique, attirent de façon non négligeable ces ménages.

C'est majoritairement dans les communes ayant adopté des politiques locales d'urbanisation privilégiant une logique résidentielle locale plus rurale que métropolitaine que l'on retrouve ce type d'habitants. Politiquement, ces communes privilégient un rapprochement avec les petites 


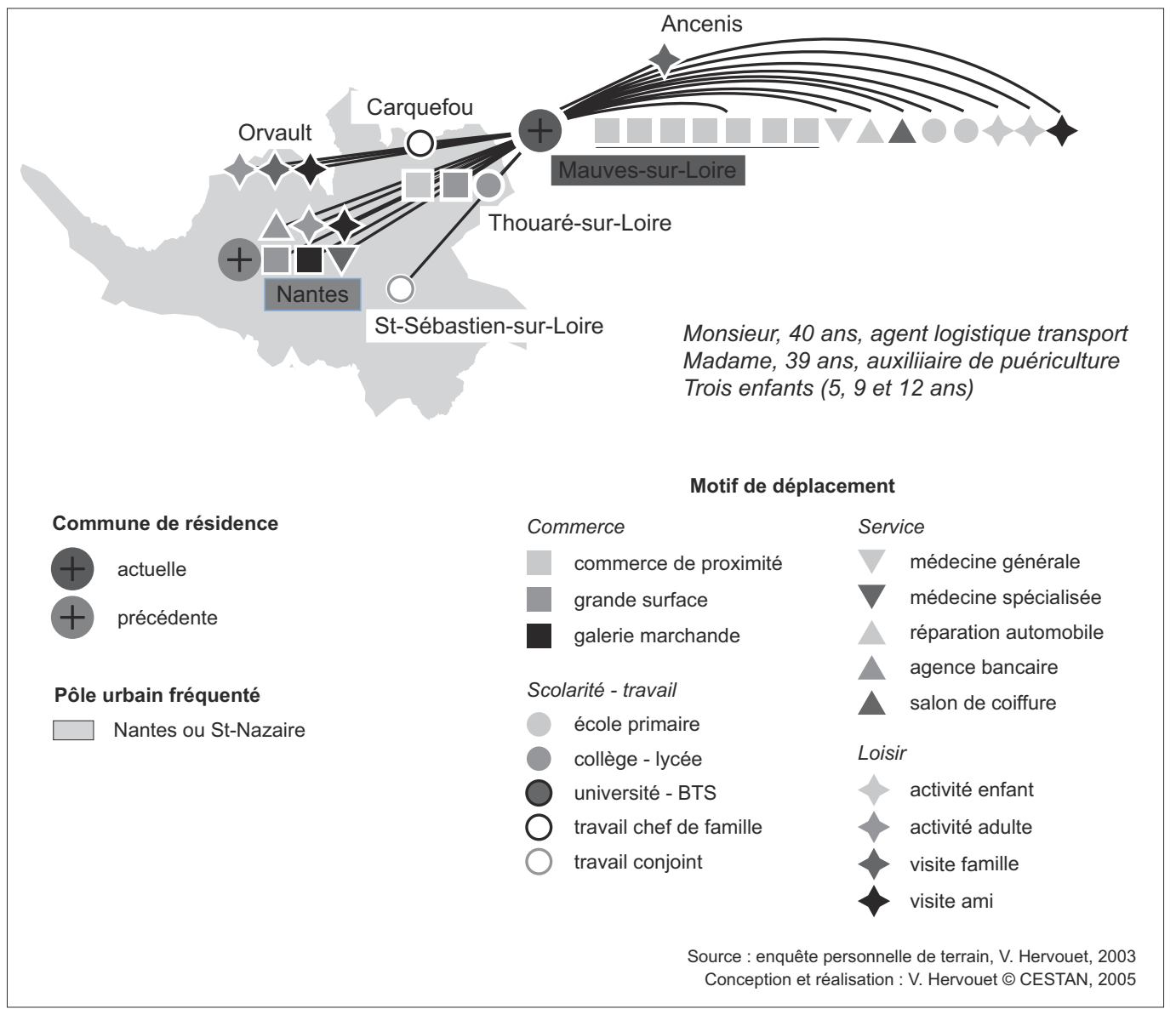

Figure 2 : Exemple de déplacements d'un ménage : le modèle du rapport grandissant à la ville An example of household mobility : the "increasing relationship with town" pattern

unités urbaines voisines plutôt que « de se faire manger par le géant urbain où [ils n'auraient] pas le droit au chapitre » (maire du Cellier, août 2003).

Mais paradoxalement, les ménages sont incités à rester très en lien avec le pôle urbain voisin s'ils veulent conserver des services (notamment commerciaux) et des activités (souvent de loisirs) dont ils jouissaient auparavant en ville : les élus locaux se montrent réticents à apporter une réponse favorable aux demandes de nouveaux équipements dans la commune (supermarché, associations, infrastructures diverses).

Les déplacements d'un ménage actif résidant à Oudon (fig. 3) reflètent parfaitement la logique intermédiaire dans laquelle se placent les individus selon ce modèle : si monsieur et madame partagent leurs déplacements de façon équilibrée entre la commune de résidence - où elle travaille - et le pôle urbain voisin - où il travaille -, ils ne répugnent pas à briser cette dichotomie en fréquentant parfois l'unité urbaine périphérique voisine - comme le supermarché de Saint-Géréon en banlieue d'Ancenis, parfois une commune proche - de la famille au Cellier - ou encore d'autres espaces périurbains - des amis et de la famille à Maisdon-sur-Sèvre, Treillières, Notre-Dame-desLandes. 


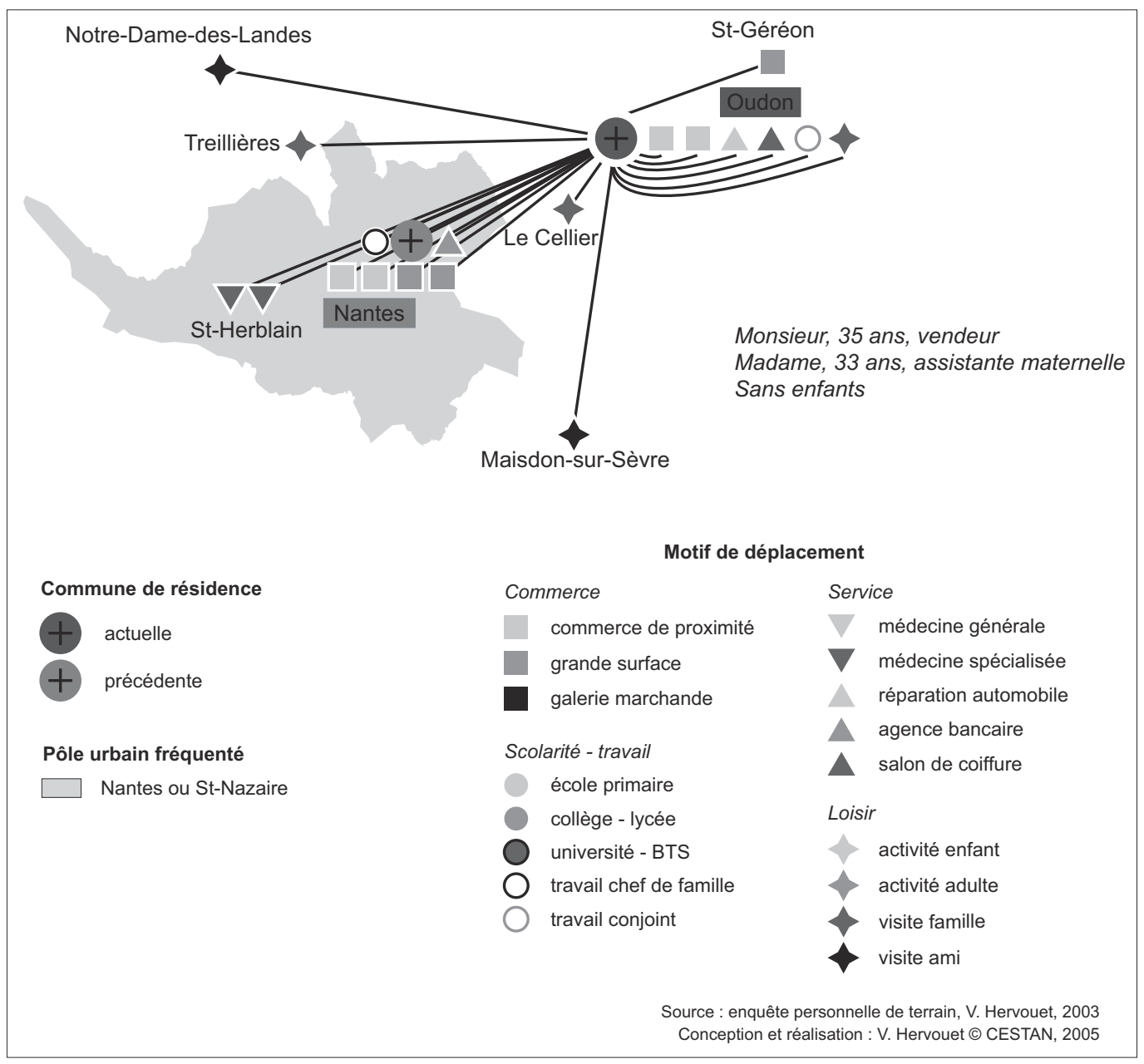

Figure 3 : Exemple de déplacements d'un ménage : le modèle du rapport pragmatique et distancié à la ville An example of household mobility : the "pragmatic and far distant relationship with town" pattern

\section{QUATRIÈME MODÈLE : UN RAPPORT RELAYÉ À LA VILLE PAR L’ÉCHELON LOCAL}

Évoluant dans un niveau d'équipement local élevé et donc offrant un premier «palier d’urbanité » pour les populations des communes environnantes moins bien pourvues, les populations sont plus facilement enclines à fréquenter la ville-centre et sa banlieue devenues alors « second palier d'urbanité ».

L'offre quotidienne élevée de commerces et de services agit comme un aiguillon pour exiger un niveau d'équipement toujours plus élevé. Habituées à une densité de population et d'habitat, ces populations fréquentent moins exceptionnellement l'agglomération voisine. Ce processus pourrait être imputable à des navettes domicile-travail centripètes et des flux résidentiels périurbains centrifuges avec le pôle urbain voisin plus nombreux qu'ailleurs; or le nombre important d'emplois locaux et un marché foncier à clientèle en partie locale infirment cette hypothèse. Par contre, la fréquentation assez importante des galeries marchandes, des agences bancaires, des lieux de loisirs pour adultes situés dans l'agglomération voisine est une marque tangible du privilège d'une urba- 
nité élevée. Les relations amicales y sont également nombreuses en plus des relations familiales, par définition moins choisies.

Cette organisation en «paliers d'urbanité » est bien illustrée par le cas d'une famille résidant à Herbignac (fig. 4). En effet, celle-ci ne fréquente que deux types de lieux : les unités urbaines périphériques et le pôle urbain voisin.

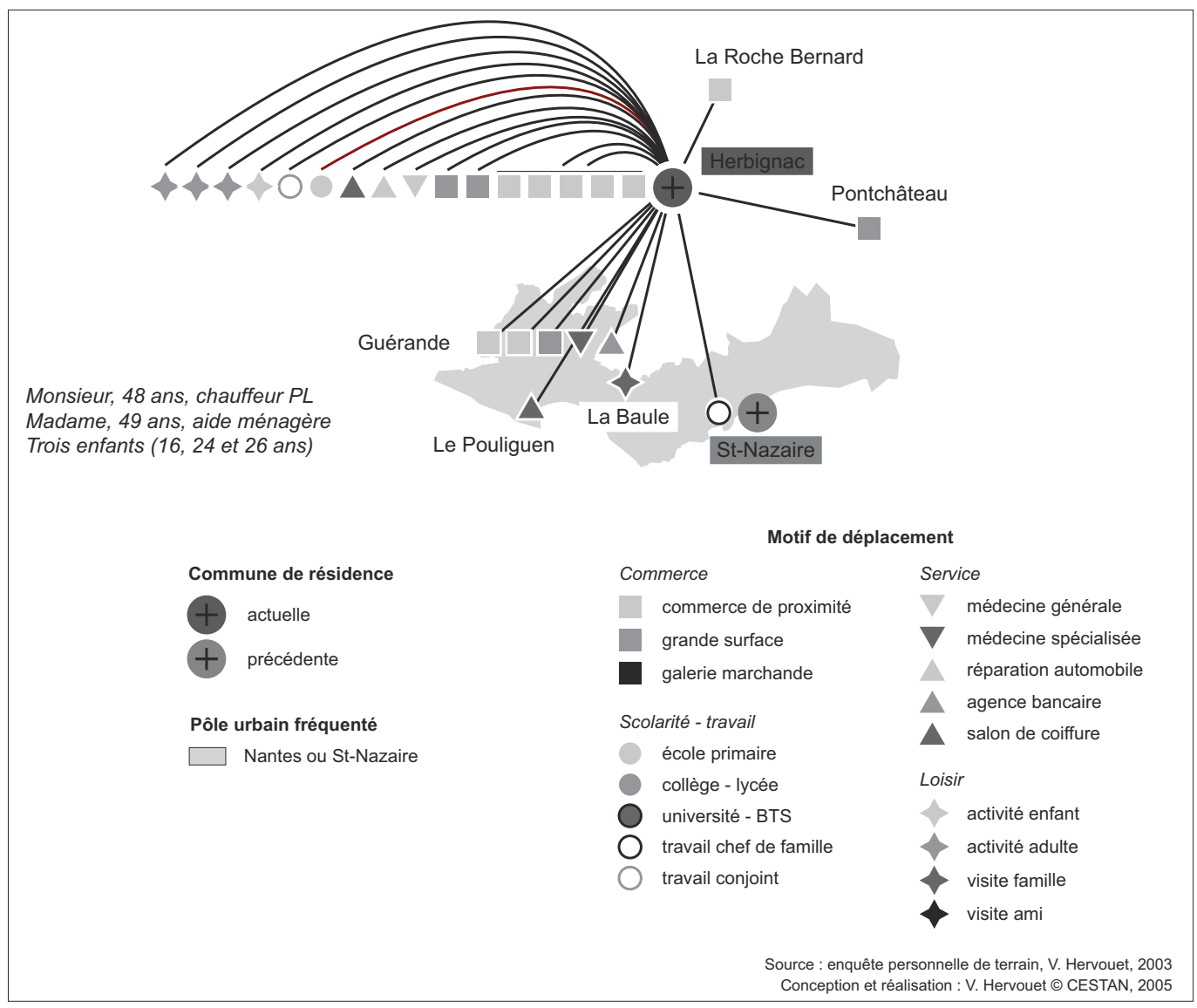

Figure 4 : Exemple de déplacements d'un ménage : le modèle du rapport relayé à la ville An example of household mobility : the "relayed relationship with town" pattern

Les unités urbaines périphériques sont constituées, bien sûr, de la commune de résidence d'Herbignac, mais aussi d'autres telles Pontchâteau ou La Roche-Bernard; le restant des migrations se réalise dans le pôle urbain nazairien : à Guérande pour son offre commerciale et médicale principalement, au Pouliguen et à La Baule pour des motifs plus ponctuels, à Saint-Nazaire pour l'emploi du chef de famille.

\section{CinquiÈme MODÈLE : UN RAPPORT CONTRAINT À LA VILLE}

Dans ce modèle, les individus ne se rendent dans l'agglomération voisine (à Nantes ou SaintNazaire par exemple) que pour des sorties rares comme des courses ponctuelles en grande surface, la fréquentation des galeries marchandes dans un but précis ou la visite d'un médecin spécialiste. 
Les liens familiaux et amicaux ne contribuent qu'assez modestement aux trajets vers l'agglomération urbaine voisine.

Le pouvoir d'attraction de la métropole grandit cependant (malgré le rejet politique et urbanistique des municipalités attachées à l'« esprit de village », qui accueillent souvent ce type de population), et les actifs sont entre un et deux cinquièmes à travailler dans la ville-centre ou dans sa banlieue. Toutefois, l'identité revendiquée par ces populations est plus souvent « rurale » ou «semi-rurale » que «semi-urbaine ».

La prééminence de la logique de fréquentation locale sur celle du pôle urbain voisin est illustrée par l'observation des déplacements d'un jeune couple avec deux enfants vivant à Saint-Hilairede-Clisson (fig. 5).

Si le faible niveau d'équipement local ne permet pas une importante fréquentation de la commune de résidence, les pérégrinations se reportent massivement sur l'unité urbaine périphérique voisine de Clisson - qui associe cette commune à Gorges et Gétigné - et sur quelques communes périurbaines voisines. À l'opposé, le pôle urbain nantais n'est fréquenté que par contrainte - pour

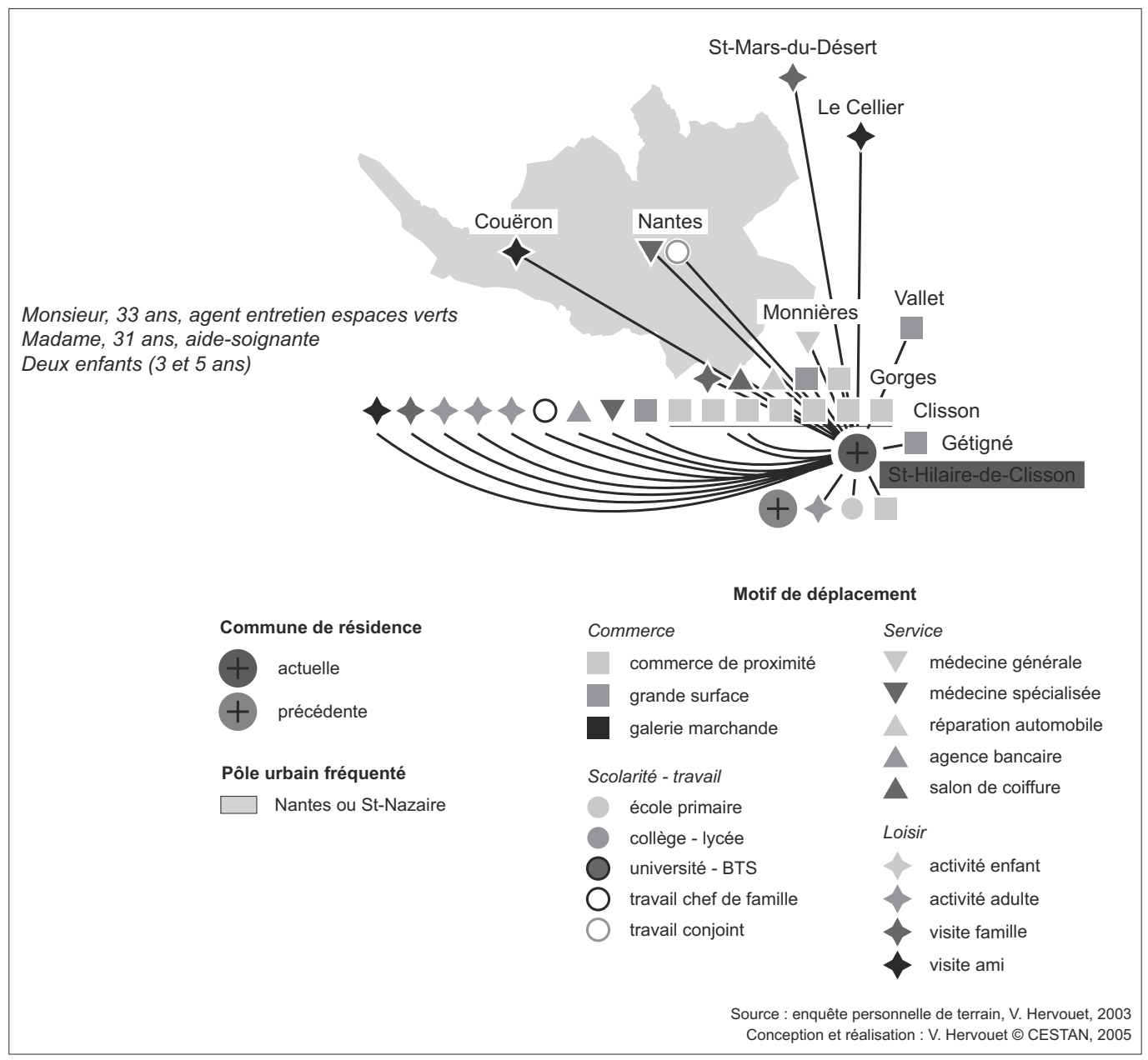

Figure 5 : Exemple de déplacements d'un ménage : le modèle du rapport contraint à la ville An example of household mobility : the "forced relationship with town" pattern 
le travail du conjoint et les médecins spécialistes - ou en de rares occasions, des amis visités deux à trois fois par an.

\section{SIXIÈME MODÈLE : UN RAPPORT NAISSANT À LA VILLE}

De façon générale, les habitudes de déplacements au quotidien des ménages de ce modèle sont en pleine mutation : si l'arrivée de nouvelles populations en provenance de l'agglomération voisine rend la présence urbaine palpable dans la commune de résidence et incite à la fréquentation de cette ville-centre et de sa banlieue, les unités urbaines voisines avec un niveau d'équipement élevé gardent un pouvoir d'attraction important sur ces populations.

Ainsi, rares sont les courses de proximité ou celles hebdomadaires en grande surface à s'effectuer au-delà d'un rayon de quinze kilomètres dans ce modèle de déplacement. Les services courants - médecine généraliste, agence bancaire, garage automobile, salon de coiffure - n'attirent que très peu ce type de populations dans le centre de la ville-centre ou dans les centres commerciaux périphériques. Même les services plus rares offerts par le pôle urbain, tels les médecins spécialistes, les galeries marchandes, ou les achats ponctuels en grande surface, restent moins polarisants pour ces individus que dans les autres modèles, y compris le précédent.

Conséquence d'un fort ancrage local des modes de vie, les attaches familiales et l'installation à la campagne sont deux critères importants avancés comme raisons d'installation dans la commune de résidence. Néanmoins, ces populations sont soumises à des marchés fonciers désormais très «tendus » que la proximité urbaine ne fera qu'exacerber à court ou moyen terme, les intégrant dans un système résidentiel métropolitain.

L’observation des déplacements d'un ménage actif résidant à Bouée (fig. 6) met en évidence d'une part, l'emprise du chef-lieu de canton voisin de Savenay sur la fréquentation et, d'autre part, la double influence déjà perceptible des pôles urbains nantais et nazairien; néanmoins ces derniers répondent seulement à des besoins rares et ponctuels malgré la localisation à Nantes du travail du chef de famille.

\section{Conclusion : quelques repères dans une formidable diversité de déplacements}

Les dynamiques spatiales et sociales des espaces périurbains sont d'une grande complexité, en raison de la multiplicité des profils personnels (au niveau des individus), des combinaisons familiales (au niveau des ménages) et des contextes résidentiels (au niveau des communes de résidence).

Toutefois, certaines récurrences peuvent être notées et c'est en ce sens que les six modèles exposés dans cet article ont été élaborés. En intégrant d'abord l'usager dans son rapport à l'automobile, tant ce moyen de locomotion tient une place primordiale dans les espaces périurbains, puis en reconsidérant ce même Homo automobilus dans ce qui fait sens pour lui et l'incite à se déplacer dans sa vie quotidienne (les facteurs structurants de l'organisation de ses déplacements), il a alors été possible de sérier des grandes formes de déplacements, différentes selon la nature des liens qu'entretiennent les populations périurbaines avec la ville-centre voisine.

Du rapport permanent au rapport plus récent à la ville, ces six modèles, attachés plus ou moins nettement à différents types de communes périurbaines (Hervouet, 2005), montrent combien la transformation des périphéries des villes est grande et combien les évolutions sont hétérogènes d'un point de vue tant social que spatial.

Au-delà des facteurs classiquement pris en compte pour étudier les espaces périurbains (dynamiques démographiques, occupation des sols, mutations politiques locales, formes d'habitat, coût financier de la périurbanisation, impacts environnementaux), cette typologie accorde une part prépondérante à la thématique majeure qui nourrit et permet l'étalement urbain, à savoir la mobilité des ménages. C'est en grande partie de l'évolution de cette mobilité dans les prochaines années, corrélée à celle du coût du carburant, que dépend la poursuite ou non de la périurbanisation. 


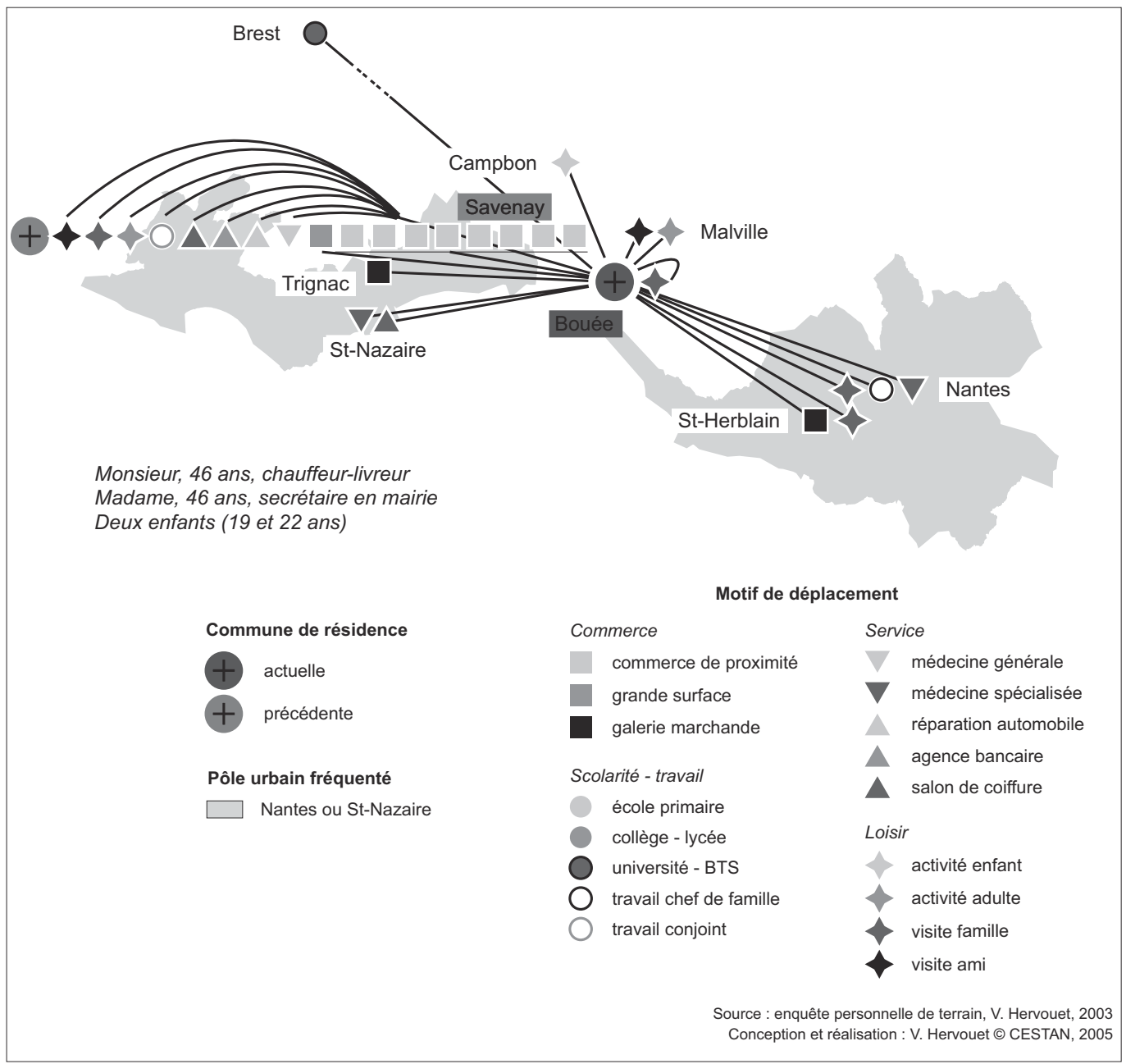

Figure 6 : Exemple de déplacements d'un ménage : le modèle du rapport naissant à la ville An example of household mobility : the "emerging relationship with town" pattern

\section{Bibliographie}

Ascher (F.), 1995. - Métapolis ou l'avenir des villes, Paris, Odile Jacob, 345 p.

Bauer (G.), Roux (J.-M.), 1976. - La rurbanisation ou la ville éparpillée, Paris, Seuil, 192 p.

Bonnet (M.), Desjeux (D.) (dir.), 2000. - Les territoires de la mobilité, Paris, PUF, coll. « Sciences sociales et société », $224 \mathrm{p}$.

Chalas (Y.), 2004. - «La réinvention de l'urbain en périphérie », Séminaire Les services publics face aux transformations de l'urbain, séance du 13 mai 2004, Toulouse Le Mirail, p. 3-9.

Dupur (G.), 1999. - La dépendance automobile : symptômes, analyses, diagnostic, traitements, Paris, Anthropos/Economica, coll. «Villes », 160 p.

Hervouet (V.), 2005. - La périurbanisatin dans la métropole nantaise, Université de Nantes, thèse de géographie et aménagement du territoire, 546 p. [http://tel.archives-ouvertes.fr/tel-0001 1626].

Jousseaume (V.), 1998. - L'ombre d'une métropole, Rennes, PUR, 209 p. 
Kaufmann (V.), Montulet (B.), 2004. - Mobilités, fluidités... libertés?, Bruxelles, facultés universitaires Saint-Louis, coll. «Travaux et Recherches », 310 p.

Orfeuil (J.-P.), 1994. - Je suis l'automobile, Paris, Éditions de l'Aube, coll. « Monde en cours », 95 p.

Pinson (D.), Thomann (S.), 2001. - La maison en ses territoires : de la villa à la ville diffuse, Paris/Budapest/ Turin, L'Harmattan, $191 \mathrm{p}$.

Rougé (L.), 2005. - Accession à la propriété et modes de vie en maison individuelle en périurbain lointain toulousain. Les « captifs » du périurbain?, thèse de géographie et aménagement du territoire, Toulouse, Université du Mirail, 381 p.

Cet article a été reçu le 16 juin 2007 et définitivement accepté le 5 novembre 2007. 